\title{
High-Flux Monochromatic Electron and Ion Beams from Laser Cooled Atoms
}

\author{
A. McCulloch ${ }^{1}$, Y. Bruneau ${ }^{1}$, G. Khalili ${ }^{1}$, D. Comparat ${ }^{1}$ \\ ${ }^{1}$ Laboratoire Aimé Cotton, CNRS, Université Paris-Sud, ENS Cachan, Bâtiment 505, 91405 Orsay, \\ France.
}

Sources of inherently cold electrons and ions have undergone rapid development in the past few years. These sources are based upon near-threshold ionization of laser cooled atoms, and show great promise for the creation of high-brightness ion beams, in particular, for use in focused ion beam systems [1, 2], as well as the creation of electron bunches suitable for ultrafast electron diffraction experiments on biological samples $[3,4]$. An aspect of the source which has yet to be exploited is the ability to produce highly monochromatic, low energy (less than $10 \mathrm{eV}$ ) electron beams, which are vital in studies of nanofunctionalization of surface compounds [5].

Due to the low electron/ion temperature associated with these sources, they possess highly desirable qualities. For example, an ion brightness of $2 \times 10^{7} \mathrm{~A} \mathrm{~m}^{-2} \mathrm{sr}^{-1} \mathrm{eV}^{-1}$ was recently reported for a $\mathrm{pA}$ current $\mathrm{Cs}^{+}$beam [1]. Likewise, cold electron beams with a source coherences of $10 \mathrm{~nm}$ have been reported, and more recently have been shown to operate in the ultrafast regime with little degradation to the beam quality [3, 4], paving the way for ultrafast diffraction experiments of weakly scattering nanocrystalline samples.

Cold electron/ion beams are typically produced via the near threshold photoionization of laser cooled atom clouds. Using a magneto-optical trap, approximately $10^{8}$ neutral atoms can be trapped with a temperature on the order of $100 \mu \mathrm{K}$ in a region measuring a few millimeters across. The cloud is then photoionized and the electrons and ions are extracted.

Here we present the realization of a cold election/ion source based not on photoionization, but rather the ionization of a beam of Rydberg atoms in a high-gradient electric field, with the aim of producing an electron beam suitable for nanofunctionalization studies. The longitudinal energy spread of a beam is fundamentally limited by the different potentials experienced by the electrons/ions upon ionization, which is in turn limited by the size of the ionization volume, which is typically on the order of $10 \mu \mathrm{m}$. Field ionization of Rydberg atoms in a high gradient field allows for a reduction of the source size [2] and hence a reduction of the source energy spread and in turn, the source brightness.

We report on the measured source properties as measured from a newly constructed apparatus and compare them to detailed simulations of the source. The applicability of the source for various applications is also discussed, and the progress toward nanofunctionalization studies is detailed.

Novel source properties are also investigated. Cold atom sources are very unique in that both electrons and ions are produced simultaneously upon ionization. The concept for a single-ion source for implantation and its realization is detailed, along with the extension of the apparatus to allow for near complete ionic trajectory correction. 


\section{References:}

[1] B. Knuffman et al, Journal of Applied Physics 114:044303 (2013).

[2] L. Kime et al, Physical Review A 88:033424 (2013).

[3] A. J. McCulloch et al, Nature Communications 4:1692 (2013).

[4] W. J. Engelen et al, Nature Communications 4:1693 (2013).

[5] A. Lafosse et al, Progress in Surface Science 84 (2009), p. 177-198.

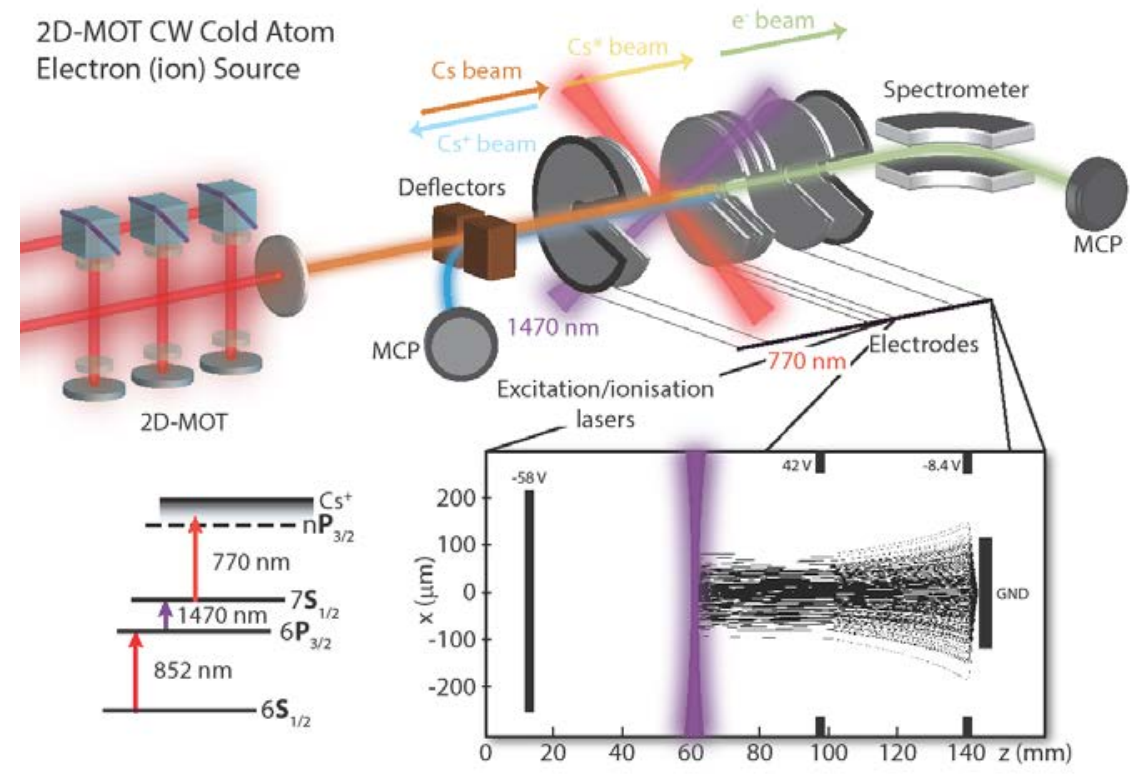

Figure 1. A schematic of the apparatus. A 2D Magneto-Optical Trap provides a low temperature neutral atom beam which is converted to a cold electron (ion) beam using a 3 step field ionisation scheme.
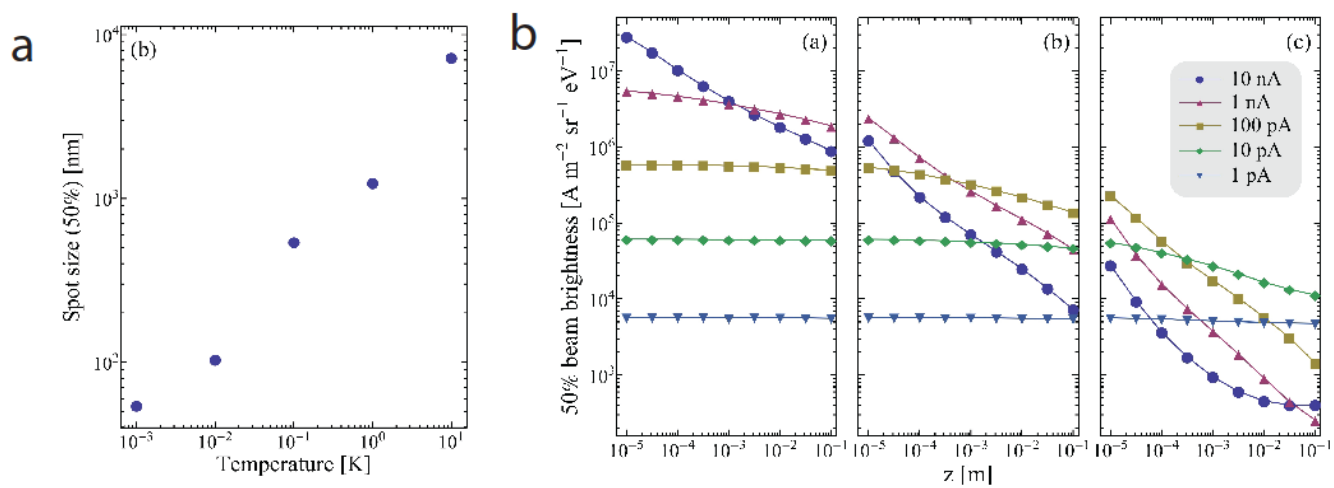

Figure 2. a) Simulations of an $8 \mathrm{eV}, 10 \mathrm{pA}$ electron beam: focal spot size versus beam temperature.

b) Simulations of ion beam brightness for versus propagation distance for $100 \mathrm{kV} / \mathrm{cm}, 10 \mathrm{kV} / \mathrm{cm}$ and 1 $\mathrm{kV} / \mathrm{cm}$ extraction fields at various currents. 